\title{
Supplement to 'TFT-Bootstrap: Resampling time series in the frequency domain to obtain replicates in the time domain'*
}

\author{
Claudia Kirch ${ }^{\dagger}$ and Dimitris N. Politis ${ }^{\ddagger}$

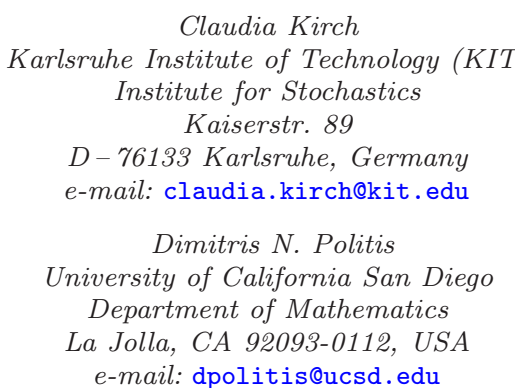

\begin{abstract}
This supplement contains the detailed proofs to the paper 'TFTBootstrap: Resampling time series in the frequency domain to obtain replicates in the time domain'. All quotations refer to this paper and the citations in this supplement refer to the list of references at the end of that paper.
\end{abstract}

AMS 2000 subject classifications: Primary 62G09; secondary 62M15, $62 \mathrm{M} 10$.

Keywords and phrases: frequency domain bootstrap, functional limit theorem, nonlinear processes, periodogram, ratio statistics, spectral density estimation, surrogate data, change-point analysis, unit root testing.

\footnotetext{
* The work was supported by the DFG graduate college 'Mathematik und Praxis'.

$\dagger$ The work was supported by the DFG graduate college 'Mathematik und Praxis' and the position of the first author was partly financed by the Stifterverband für die Deutsche Wissenschaft by funds of the Claussen-Simon-trust.

¥The research was partially supported by NSF Grant DMS-07-06732.
} 


\section{Appendix A: Proofs of Section 3}

We start with a short lemma needed to prove Lemma 3.1.

Lemma A.1. Under Assumption P.1, the following representation holds

$$
\frac{4 \pi}{T} \sum_{k=1}^{N} f\left(\lambda_{k}\right) \cos \left(\lambda_{k} h\right)=\sum_{l \in \mathbb{Z}} \gamma(h+l T)-\frac{2 \pi}{T} f(0)-\frac{2 \pi}{T} f(\pi) \exp (i \pi h) 1_{\{T \text { even }\}},
$$

$N=\lfloor(T-1) / 2\rfloor, \lambda_{k}=2 \pi k / T$.

Proof. First it holds (cf. e.g. Brockwell and Davis [5], Corollary 4.3.2)

$$
f(\lambda)=\frac{1}{2 \pi} \sum_{j=-\infty}^{\infty} \gamma(j) \exp (-i j \lambda)
$$

Since $\cos \left(\lambda_{k} h\right)=\frac{1}{2}\left(\exp \left(i h \lambda_{k}\right)+\exp \left(i h \lambda_{T-k}\right)\right)$, we obtain

$$
\begin{aligned}
& \frac{4 \pi}{T} \sum_{k=1}^{N} f\left(\lambda_{k}\right) \cos \left(\lambda_{k} h\right) \\
& =\frac{2 \pi}{T} \sum_{k=0}^{T-1} f\left(\lambda_{k}\right) \exp \left(i h \lambda_{k}\right)-\frac{2 \pi}{T} f(0)-\frac{2 \pi}{T} f(\pi) \exp (i \pi h) 1_{\{T \text { even }\}} .
\end{aligned}
$$

Moreover

$$
\begin{aligned}
& \frac{2 \pi}{T} \sum_{k=0}^{T-1} f\left(\lambda_{k}\right) \exp \left(i h \lambda_{k}\right)=\frac{1}{T} \sum_{k=0}^{T-1} \sum_{j=-\infty}^{\infty} \gamma(j) \exp \left(-i j \lambda_{k}\right) \exp \left(i h \lambda_{k}\right) \\
& =\frac{1}{T} \sum_{j=-\infty}^{\infty} \gamma(j) \sum_{k=0}^{T-1} \exp \left(i(h-j) \lambda_{k}\right)=\sum_{l \in \mathbb{Z}} \gamma(h+l T),
\end{aligned}
$$

where we can switch the two sums because of Fubini's theorem.

We are now ready to prove Lemma 3.1.

Proof of Lemma 3.1. Assertion a) follows immediately from the fact that the bootstrapped Fourier coefficients are conditionally centered. By Lemma A.4 in Kirch [31] it holds (uniformly in $u$ )

$$
\sum_{l=1}^{\lfloor m u\rfloor} \cos \left(\lambda_{k} l\right)=O\left(\min \left(\frac{T}{k}, m\right)\right)
$$

and the same expression for sine instead of cosine. Thus it holds uniformly in $u$ and $v$

$$
\sum_{k=1}^{N}\left|\sum_{l_{1}=1}^{\lfloor m u\rfloor} \cos \left(\lambda_{k} l_{1}\right)\right|\left|\sum_{l_{2}=1}^{\lfloor m v\rfloor} \cos \left(\lambda_{k} l_{2}\right)\right|=O(1) \sum_{k=1}^{N} \min \left(\frac{T}{k}, m\right)^{2}=O(m T) .
$$


The same equation holds true if we replace cosine by sine.

By Assumptions $\mathcal{B} .1$ and $\mathcal{B} .2$ and by $(2.4)$ it holds

$$
\begin{aligned}
& \operatorname{cov}^{*}\left(\frac{1}{\sqrt{m}} \sum_{l_{1}=1}^{\lfloor m u\rfloor} Z^{*}\left(l_{1}\right), \frac{1}{\sqrt{m}} \sum_{l_{2}=1}^{\lfloor m v\rfloor} Z^{*}\left(l_{2}\right)\right) \\
& =\frac{4}{T m} \sum_{l_{1}=1}^{\lfloor m u\rfloor} \sum_{l_{2}=1}^{\lfloor m v\rfloor} \sum_{k=1}^{N} \operatorname{var}^{*}\left(x^{*}(k)\right) \cos \left(l_{1} \lambda_{k}\right) \cos \left(l_{2} \lambda_{k}\right) \\
& +\frac{4}{T m} \sum_{l_{1}=1}^{\lfloor m u\rfloor} \sum_{l_{2}=1}^{\lfloor m v\rfloor} \sum_{k=1}^{N} \operatorname{var}^{*}\left(y^{*}(k)\right) \sin \left(l_{1} \lambda_{k}\right) \sin \left(l_{2} \lambda_{k}\right) \\
& =\frac{4 \pi}{T m} \sum_{l_{1}=1}^{\lfloor m u\rfloor} \sum_{l_{2}=1}^{\lfloor m v\rfloor} \sum_{k=1}^{N} f\left(\lambda_{k}\right) \cos \left(\lambda_{k}\left(l_{1}-l_{2}\right)\right)+o_{P}(1),
\end{aligned}
$$

where the last line follows for $m / T \rightarrow 0$ as well as $m=T$ by (A.1). We will now use Lemma A.1. W.l.o.g. let $u \leqslant v$. Summing the first term of Lemma A.1 we have, e.g. by the proof of Corollary 4.3.2 in Brockwell and Davis [5], which gives the relationship between the autocovariance function and the spectral density,

$$
\begin{aligned}
& \frac{1}{m} \sum_{l_{1}=1}^{\lfloor m u\rfloor} \sum_{l_{2}=1}^{\lfloor m v\rfloor} \gamma\left(l_{1}-l_{2}\right) \\
& =\frac{1}{m} \sum_{l_{1}=1}^{\lfloor m u\rfloor} \sum_{l_{2}=1}^{\lfloor m u\rfloor} \gamma\left(l_{1}-l_{2}\right)+\frac{1}{m} \sum_{l_{1}=1}^{\lfloor m u\rfloor} \sum_{l_{2}=\lfloor m u\rfloor+1}^{\lfloor m v\rfloor} \gamma\left(l_{1}-l_{2}\right) \\
& =2 \pi f(0) u+o(1),
\end{aligned}
$$

since by the absolute summability of $\gamma(\cdot)$

$$
\frac{1}{m} \sum_{l_{1}=1}^{\lfloor m u\rfloor} \sum_{l_{2}=\lfloor m u\rfloor+1}^{\lfloor m v\rfloor}\left|\gamma\left(l_{2}-l_{1}\right)\right| \leqslant \frac{1}{m} \sum_{h=1}^{\lfloor m v\rfloor-1} h|\gamma(h)|=o(1) .
$$

Furthermore

$$
\begin{aligned}
& \frac{1}{m} \sum_{l_{1}=1}^{\lfloor m u\rfloor} \sum_{l_{2}=1}^{\lfloor m v\rfloor} \sum_{j \neq 0}\left|\gamma\left(l_{1}-l_{2}+j T\right)\right| \leqslant \sum_{|h| \leqslant m} \frac{m-|h|}{m} \sum_{j \neq 0}|\gamma(h+j T)| \\
& \leqslant \frac{2}{\sqrt{m}} \sum_{j \in \mathbb{Z}}|\gamma(j)|+\sum_{|h| \leqslant m-\sqrt{m}} \sum_{j \neq 0}|\gamma(h+j T)|=o(1)+2 \sum_{k \geqslant \sqrt{m}}|\gamma(k)|=o(1) .
\end{aligned}
$$

Summing the last two terms of Lemma A.1 we obtain

$$
\frac{1}{T m} \sum_{l_{1}} \sum_{l_{2}} \exp \left(i \pi\left(l_{1}-l_{2}\right)\right)=\frac{1}{T m} \sum_{l_{1}} \exp \left(\pi i l_{1}\right) \sum_{l_{2}} \exp \left(-\pi i l_{2}\right)=O\left(\frac{1}{T m}\right)
$$


and

$$
2 \pi f(0) \frac{1}{T m}(\lfloor m v\rfloor)(\lfloor m u\rfloor)= \begin{cases}o(1), & \frac{m}{T} \rightarrow 0, \\ 2 \pi f(0) u v+o(1), & m=T .\end{cases}
$$

Putting everything together we obtain b). The proof of c) is analogous. A simple calculation shows that $\operatorname{cov}\left(Z\left(l_{1}\right), Z\left(l_{2}\right)\right)=\operatorname{cov}\left(V\left(l_{1}\right), V\left(l_{2}\right)\right)+o(1)$ by the absolute summability of the auto-covariance function.

The next lemma gives the crucial step towards tightness of the partial sum process.

Lemma A.2. Under Assumptions P.1, B.1 - B.3 it holds for $u<v$

$$
\mathrm{E}^{*}\left(\frac{1}{\sqrt{m}} \sum_{l=\lfloor m u\rfloor+1}^{\lfloor m v\rfloor} Z^{*}(l)\right)^{4} \leqslant\left(D+o_{P}(1)\right)(v-u)^{2}
$$

for some constant $D>0$.

Proof. Note that for a sum of independent random variables with mean zero it holds

$$
\mathrm{E}\left(\sum_{k} X_{k}\right)^{4}=\sum_{k} \mathrm{E} X_{k}^{4}+6\left(\sum_{k} \mathrm{E} X_{k}^{2}\right)^{2}-6 \sum_{k}\left(\mathrm{E} X_{k}^{2}\right)^{2} .
$$

Furthermore

$$
\begin{aligned}
& \frac{1}{\sqrt{m}} \sum_{l} Z^{*}(l) \\
& =\frac{2}{\sqrt{T m}} \sum_{k=1}^{N}\left(x^{*}(k) \sum_{l} \cos \left(\lambda_{k} l\right)-y^{*}(k) \sum_{l} \sin \left(\lambda_{k} l\right)\right)=: \frac{1}{\sqrt{T m}} \sum_{k=1}^{2 N} Y_{k}^{*},
\end{aligned}
$$

where $Y_{k}^{*}=x^{*}(k) \sum_{l} \cos \left(\lambda_{k} l\right)$ and $Y_{N+k}^{*}=-y^{*}(k) \sum_{l} \sin \left(\lambda_{k} l\right)$ for $k \leqslant N$. Thus, we will verify the assumption of the lemma for all three summands of eq. (A.2).

First it holds similarly to (A.1) by Assumption $\mathcal{B} .2$ and $\mathcal{B} .3$

$$
\begin{aligned}
& \frac{1}{m^{2} T^{2}} \sum_{k=1}^{2 N} \mathrm{E}^{*}\left(Y_{k}^{*}\right)^{4} \\
& \leqslant\left(C+o_{P}(1)\right) \frac{1}{m^{2} T^{2}} \sum_{k} \min \left(\frac{T}{k}, m(v-u)\right)^{4} \leqslant\left(D_{1}+o_{P}(1)\right)(v-u)^{2} .
\end{aligned}
$$


Secondly we have by Assumption $\mathcal{B} .2$ and Lemma A.1 similarly to (A.1)

$$
\begin{aligned}
& \frac{1}{m T} \sum_{k} \mathrm{E}^{*}\left(Y_{k}^{*}\right)^{2} \\
& =\frac{4 \pi}{m T} \sum_{l_{1}=\lfloor m u\rfloor+1}^{\lfloor m v\rfloor} \sum_{l_{2}=\lfloor m u\rfloor+1}^{\lfloor m v\rfloor} \sum_{k} f\left(\lambda_{k}\right) \cos \left(\lambda_{k}\left(l_{1}-l_{2}\right)\right)+o_{P}(1)(v-u) \\
& =\frac{1}{m} \sum_{l_{1}=\lfloor m u\rfloor+1}^{\lfloor m v\rfloor} \sum_{l_{2}=\lfloor m u\rfloor+1}^{\lfloor m v\rfloor} \sum_{j \in \mathbb{Z}} \gamma\left(l_{2}-l_{1}+j T\right)+O(1)(v-u)^{2}+o_{P}(1)(v-u) \\
& \leqslant\left(D_{2}+o_{P}(1)\right)(v-u),
\end{aligned}
$$

since

$$
\begin{aligned}
& \frac{1}{m} \sum_{l_{1}=\lfloor m u\rfloor+1}^{\lfloor m v\rfloor} \sum_{l_{2}=\lfloor m u\rfloor+1}^{\lfloor m v\rfloor} \sum_{j \in \mathbb{Z}}\left|\gamma\left(l_{2}-l_{1}+j T\right)\right| \\
& =\sum_{|h|<\lfloor m v\rfloor-\lfloor m u\rfloor} \frac{\lfloor m v\rfloor-\lfloor m u\rfloor-|h|}{m} \sum_{j \in \mathbb{Z}}|\gamma(h+j T)| \leqslant 2(v-u) \sum_{k \in \mathbb{Z}}|\gamma(k)| .
\end{aligned}
$$

Finally it holds

$$
\begin{aligned}
& \frac{1}{m^{2} T^{2}} \sum_{k}\left(\mathrm{E}^{*}\left(Y_{k}^{*}\right)^{2}\right)^{2} \\
& =\frac{1}{m^{2} T^{2}} \sum_{k=1}^{N}\left(\left(\pi f\left(\lambda_{k}\right)+o_{P}(1)\right) \sum_{l_{1}, l_{2}}\left(\cos \left(\lambda_{k} l_{2}\right) \cos \left(\lambda_{k} l_{1}\right)+\sin \left(\lambda_{k} l_{2}\right) \sin \left(\lambda_{k} l_{1}\right)\right)\right)^{2} \\
& \leqslant\left(D_{3}+o_{P}(1)\right) \frac{1}{m^{2} T^{2}} \sum_{k} \max \left(\frac{T}{k}, m(v-u)\right)^{4} \leqslant\left(D_{4}+o_{P}(1)\right)(v-u)^{2}
\end{aligned}
$$

The next lemma gives the convergence of the finite-dimensional distribution.

Lemma A.3. Let $S_{m}^{*}(u)=\frac{1}{\sqrt{m}} \sum_{j=1}^{\lfloor m u\rfloor} Z^{*}(j)$.

a) If Assumptions P.1, B.1 - B.3 are fulfilled and $m / T \rightarrow 0$ we obtain for all $0<u_{1}, \ldots, u_{p} \leqslant 1$ in probability

$$
\left(S_{m}^{*}\left(u_{1}\right), \ldots, S_{m}^{*}\left(u_{p}\right)\right) \stackrel{\mathcal{L}}{\longrightarrow} N(0, \Sigma),
$$

where $\Sigma=\left(c_{i, j}\right)_{i, j=1, \ldots, p}$ with $c_{i, j}=2 \pi f(0) \min \left(u_{i}, u_{j}\right)$.

b) If Assumptions P. $1, \mathcal{B} .1$ and $\mathcal{B} .4$ are fulfilled we obtain for all $0<u_{1}, \ldots, u_{p} \leqslant$ 1 in probability

$$
\left(S_{T}^{*}\left(u_{1}\right), \ldots, S_{T}^{*}\left(u_{p}\right)\right) \stackrel{\mathcal{L}}{\longrightarrow} N(0, \Sigma),
$$

where $\Sigma=\left(c_{i, j}\right)_{i, j=1, \ldots, p}$ with $c_{i, j}=2 \pi f(0)\left(\min \left(u_{i}, u_{j}\right)-u_{i} u_{j}\right)$. 
Proof. For the assertion in a) we use the Cramer Wold device and prove a Lyapunov type condition. Let $\alpha_{i} \in \mathbb{R}$ and consider

$$
\begin{aligned}
& \sum_{i=1}^{p} \alpha_{i} S_{m}^{*}\left(u_{i}\right) \\
& =\frac{1}{\sqrt{m T}} \sum_{k=1}^{N} 2\left[x^{*}(k) \sum_{i=1}^{p} \alpha_{i} \sum_{l=1}^{\left\lfloor m u_{i}\right\rfloor} \cos \left(\lambda_{k} l\right)+y^{*}(k) \sum_{i=1}^{p} \alpha_{i} \sum_{l=1}^{\left\lfloor m u_{i}\right\rfloor} \sin \left(\lambda_{k} l\right)\right] \\
& =: \frac{1}{\sqrt{m T}} \sum_{k=1}^{2 N} \tilde{Y}_{k, N}^{*},
\end{aligned}
$$

where $\left\{\tilde{Y}_{k, N}^{*}: 1 \leqslant k \leqslant 2 N\right\}$ is conditionally row-wise independent. The Lyapunov condition is then (in probability) fulfilled since by Assumption $\mathcal{B} .2$ and $\mathcal{B} .3$ similarly to $(\mathrm{A} .1)$

$$
\begin{aligned}
& \frac{1}{m^{2} T^{2}} \sum_{k=1}^{2 N} \mathrm{E}^{*}\left(\tilde{Y}_{k, N}^{*}-\mathrm{E}^{*} \tilde{Y}_{k, N}^{*}\right)^{4} \leqslant\left(C+o_{P}(1)\right) \frac{1}{m^{2} T^{2}} \sum_{k=1}^{N} \max \left(\frac{T}{k}, m\right)^{4} \\
& \leqslant\left(C+o_{P}(1)\right) \frac{m}{T}=o_{P}(1)
\end{aligned}
$$

Together with Lemma 3.1 this gives assertion a). Note that it is essential that $m / T \rightarrow 0$, in fact it is easy to see that for $m=T$ the Feller condition is not fulfilled, thus the Lindeberg condition can also not be fulfilled.

Therefore we need a different argument to obtain asymptotic normality for $m=T$. We will use here somewhat stronger assumptions but it is not clear, whether they are really necessary (cf. also Remark 3.2). We use now the Cramer Wold device and Lemma 3 in Mallows [38], which gives an upper bound for the Mallows distance of weighted sums of independent random variables with standard normal random variables. The assertion then follows, since by the proof of Lemma 3.1

$$
\begin{aligned}
& \sum_{i=1}^{p} \sum_{j=1}^{p} \alpha_{i} \alpha_{j} \frac{1}{T^{2}} \sum_{k=1}^{N} f\left(\lambda_{k}\right) \sum_{l_{1}=1}^{\left\lfloor T u_{i}\right\rfloor} \sum_{l_{2}=1}^{\left\lfloor T u_{j}\right\rfloor} \cos \left(\lambda_{k}\left(l_{2}-l_{1}\right)\right) \\
& =\sum_{i=1}^{p} \sum_{j=1}^{p} \alpha_{i} \alpha_{j}\left(\min \left(u_{i}, u_{j}\right)-u_{i} u_{j}\right)+o_{P}(1) .
\end{aligned}
$$

Proof of Corollary 3.2. Is analogous to the proof of Lemma A.3 a) above.

We are now ready to prove the main theorem.

Proof of Theorem 3.1. Billingsley [2], Theorem 13.5, gives a characterization of weak convergence via convergence of the finite-dimensional distributions as well 
as tightness, which can be obtained by moment conditions. Lemmas A.2 and A.3 show that these conditions are fulfilled and thus imply

$$
\begin{gathered}
\left\{\frac{1}{\sqrt{m}} \sum_{l=1}^{\lfloor m u\rfloor}\left(Z^{*}(l)-\mathrm{E}^{*} Z^{*}(l)\right): 0 \leqslant u \leqslant 1\right\} \\
\stackrel{[0,1]}{\longrightarrow} \begin{cases}\{W(u): 0 \leqslant u \leqslant 1\}, & \frac{m}{T} \rightarrow 0, \\
\{B(u): 0 \leqslant u \leqslant 1\}, & m=T .\end{cases}
\end{gathered}
$$

\section{Appendix B: Proofs of Section 4}

We introduce the notation $a_{n} \preceq b_{n}: \Leftrightarrow a_{n}=O\left(b_{n}\right)$.

Proof of Theorem 4.1. The assertion of a) follows directly from the definition of the bootstrap schemes. In the following we only prove the assertions for $x^{*}(\cdot)$, the assertions for $y^{*}(\cdot)$ follow because $x^{*}(j) \stackrel{\mathcal{L}}{=} y^{*}(j)$ (conditionally given $V(\cdot)$ ).

\section{b) Residual-Based Bootstrap RB}

By Assumption $\mathcal{A} .1$ we have

$$
\sup _{k}\left|\operatorname{var}^{*}\left(x^{*}(k)\right)-\pi f\left(\lambda_{k}\right)\right|=\sup _{k}\left|\pi \widehat{f}\left(\lambda_{k}\right)-\pi f\left(\lambda_{k}\right)\right| \stackrel{P}{\longrightarrow} 0,
$$

thus (i). Moreover concerning (ii) it holds

$$
\begin{aligned}
& \sup _{k} \mathrm{E}^{*}\left(x^{*}(k)\right)^{4} \\
& =\sup _{k}\left(\pi^{2} \widehat{f}^{2}\left(\lambda_{k}\right)\right) \frac{1}{2 N} \sum_{j=1}^{2 N}\left(\widetilde{s}_{j}-\frac{1}{2 N} \sum_{l=1}^{2 N} \widetilde{s}_{l}\right)^{4}\left(\frac{1}{2 N} \sum_{j=1}^{2 N}\left(\widetilde{s}_{j}-\frac{1}{2 N} \sum_{k=1}^{2 N} \widetilde{s}_{k}\right)^{2}\right)^{-2} \\
& \leqslant C+o_{P}(1)
\end{aligned}
$$


since by Assumption $\mathcal{A} .2$

$$
\begin{aligned}
& \frac{1}{2 N} \sum_{j=1}^{2 N}\left(\widetilde{s}_{j}-\frac{1}{2 N} \sum_{k=1}^{2 N} \widetilde{s}_{k}\right)^{2}=\frac{1}{2 N} \sum_{j=1}^{N} \frac{I(j)}{\pi \widehat{f}\left(\lambda_{j}\right)}-\left(\frac{1}{2 N} \sum_{j=1}^{N} \frac{x(j)+y(j)}{\sqrt{\pi \widehat{f}\left(\lambda_{j}\right)}}\right)^{2} \\
& =\frac{1}{2 N} \sum_{j=1}^{N} \frac{I(j)}{\pi f\left(\lambda_{j}\right)}-\left(\frac{1}{2 N} \sum_{j=1}^{N} \frac{x(j)+y(j)}{\sqrt{\pi f\left(\lambda_{j}\right)}}\right)^{2} \\
& \quad+O(1) \sup _{k}\left|\frac{f\left(\lambda_{k}\right)-\widehat{f}\left(\lambda_{k}\right)}{\pi \widehat{f}\left(\lambda_{k}\right)}\right| \frac{1}{N} \sum_{j=1}^{N} \frac{I(j)}{f\left(\lambda_{j}\right)} \stackrel{P}{\longrightarrow} 1, \\
& \frac{1}{2 N} \sum_{j=1}^{2 N} \widetilde{s}_{j}^{4} \leqslant \frac{1}{2 N} \sum_{j=1}^{N} \frac{I(j)^{2}}{\left(\pi f\left(\lambda_{j}\right)\right)^{2}}\left(1+\sup _{k}\left(\frac{f\left(\lambda_{k}\right)-\widehat{f}\left(\lambda_{k}\right)}{\pi \widehat{f}\left(\lambda_{k}\right)}\right)^{2}\right) \leqslant C+o_{P}(1) .
\end{aligned}
$$

Finally we prove (iii). Let $\left\{U_{N}(j): 1 \leqslant j \leqslant 2 N\right\}$ be i.i.d. taking the values $1, \ldots, 2 N$ with equal probability. Denote $\widetilde{s}_{j}^{*}=\widetilde{s}_{U_{N}(j)}$ (i.i.d.),

$\widetilde{\widetilde{s}}_{j}^{*}=\sqrt{\widehat{f}\left(\lambda_{U_{N}(j)}\right) / f\left(\lambda_{U_{N}(j)}\right)} \widetilde{s}_{U_{N}(j)}$ (i.i.d) and $s_{j}^{*}=s_{U_{N}(j)}$ (i.i.d.), furthermore

$x^{*}(j) \stackrel{\mathcal{L}^{*}}{=} \sqrt{\pi \widehat{f}\left(\lambda_{j}\right)} s_{j}^{*}, y^{*}(j) \stackrel{\mathcal{L}^{*}}{=} \sqrt{\pi \widehat{f}\left(\lambda_{j}\right)} s_{N+j}^{*}, j=1 \ldots, N$. Similarly to (B.1) we get

$$
\begin{aligned}
& \mathrm{E}^{*}\left(s_{1}^{*}\right)^{2}=1, \\
& \begin{aligned}
& \mathrm{E}^{*}\left(s_{1}^{*}-\widetilde{s}_{1}^{*}\right)^{2} \preceq \frac{\left|\frac{1}{2 N} \sum_{j=1}^{2 N}\left(\widetilde{s}_{j}-\frac{1}{2 N} \sum_{k=1}^{2 N} \widetilde{s}_{k}\right)^{2}-1\right|}{\frac{1}{2 N} \sum_{j=1}^{2 N}\left(\widetilde{s}_{j}-\frac{1}{2 N} \sum_{k=1}^{2 N} \widetilde{s}_{k}\right)^{2}} \frac{1}{2 N} \sum_{j=1}^{2 N} \widetilde{s}_{j}^{2} \\
&+\frac{\left(\frac{1}{2 N} \sum_{j=1}^{2 N} \widetilde{s}_{j}\right)^{2}}{\frac{1}{2 N} \sum_{j=1}^{2 N}\left(\widetilde{s}_{j}-\frac{1}{2 N} \sum_{k=1}^{2 N} \widetilde{s}_{k}\right)^{2}}=o_{P}(1), \\
& \mathrm{E}^{*}\left(\widetilde{s}_{1}^{*}-\widetilde{s}_{1}^{*}\right)^{2} \preceq \sup _{1 \leqslant l \leqslant N} \frac{\left|f\left(\lambda_{l}\right)-\widehat{f}\left(\lambda_{l}\right)\right|}{f\left(\lambda_{l}\right)} \frac{1}{2 N} \sum_{j=1}^{2 N} \widetilde{s}_{j}^{2}=o_{P}(1) .
\end{aligned}
\end{aligned}
$$


From this and Assumption $\mathcal{A} .1$ it follows

$$
\begin{aligned}
& \sup _{1 \leqslant j \leqslant N} d_{2}^{2}\left(\mathcal{L}^{*}\left(x^{*}(j)\right), N\left(0, \pi f\left(\lambda_{j}\right)\right)\right) \\
& \preceq \sup _{1 \leqslant j \leqslant N} d_{2}^{2}\left(\mathcal{L}^{*}\left(x^{*}(j)\right), \mathcal{L}^{*}\left(\sqrt{\pi f\left(\lambda_{j}\right)} s_{j}^{*}\right)\right) \\
& \quad+\sup _{1 \leqslant j \leqslant N} d_{2}^{2}\left(\mathcal{L}^{*}\left(\sqrt{\pi f\left(\lambda_{j}\right)} s_{j}^{*}\right), N\left(0, \pi f\left(\lambda_{j}\right)\right)\right) \\
& \leqslant \pi \sup _{j}\left|f\left(\lambda_{j}\right)-\widehat{f}\left(\lambda_{j}\right)\right| \mathrm{E}^{*}\left(s_{1}^{*}\right)^{2}+\pi \sup _{j}\left|f\left(\lambda_{j}\right)\right| d_{2}^{2}\left(\mathcal{L}^{*}\left(s_{1}^{*}\right), N(0,1)\right) \\
& \preceq o_{P}(1)+d_{2}^{2}\left(\mathcal{L}^{*}\left(s_{1}^{*}\right), \mathcal{L}^{*}\left(\widetilde{s}_{1}^{*}\right)\right)+d_{2}^{2}\left(\mathcal{L}^{*}\left(\widetilde{s}_{1}^{*}\right), \mathcal{L}^{*}\left(\widetilde{\widetilde{s}}_{1}^{*}\right)\right)+d_{2}^{2}\left(\mathcal{L}^{*}\left(\widetilde{\widetilde{s}}_{1}^{*}\right), N(0,1)\right) \\
& \preceq o_{P}(1)+\mathrm{E}^{*}\left(s_{1}^{*}-\widetilde{s}_{1}^{*}\right)^{2}+\mathrm{E}^{*}\left(\widetilde{s}_{1}^{*}-\widetilde{\widetilde{s}}_{1}^{*}\right)^{2}+d_{2}^{2}\left(\mathcal{L}^{*}\left(\widetilde{\widetilde{s}}_{1}^{*}\right), N(0,1)\right) \preceq o(1),
\end{aligned}
$$

where the last line follows since $\mathcal{L}^{*}\left(\widetilde{\widetilde{s}}_{1}^{*}\right)$ (conditionally on $V(\cdot)$ ) is given by the (empirical) distribution in Assumption $\mathcal{A} .3$ and by Assumption $\mathcal{A} .2$ we have the correct convergence of the first and second moment, which together gives convergence in the Mallows distance.

c) Wild Bootstrap WB

Concerning $\mathcal{B} .2$ it holds by Assumption $\mathcal{A} .1$

$$
\sup _{k}\left|\operatorname{var}^{*}\left(x^{*}(k)\right)-\pi f\left(\lambda_{k}\right)\right|=\sup _{k}\left|\pi \widehat{f}\left(\lambda_{k}\right)-\pi f\left(\lambda_{k}\right)\right| \stackrel{P}{\longrightarrow} 0 .
$$

Similarly we obtain $\mathcal{B} .3$ since

$$
\sup _{k} \mathrm{E}^{*}\left(x^{*}(k)\right)^{4}=3 \pi^{2} \sup _{k} \widehat{f}\left(\lambda_{k}\right)^{2} \leqslant 3 \pi^{2} \sup _{k} f\left(\lambda_{k}\right)^{2}+o_{P}(1) \leqslant C+o_{P}(1) \text {. }
$$

Concerning $\mathcal{B} .4$ let $X \stackrel{\mathcal{L}}{=} N(0,1)$, then $\sqrt{\pi \widehat{f}\left(\lambda_{k}\right)} X \stackrel{\mathcal{L}^{*}}{=} x^{*}(k)$. Then

$$
\begin{aligned}
& \sup _{k} d_{2}^{2}\left(\mathcal{L}^{*}\left(x^{*}(k)\right), N\left(0, \pi f\left(\lambda_{k}\right)\right)\right) \leqslant \pi \sup _{k}\left(\sqrt{f\left(\lambda_{k}\right)}-\sqrt{\widehat{f}\left(\lambda_{k}\right)}\right)^{2} \mathrm{E} X^{2} \\
& \leqslant \pi \sup _{k}\left|\widehat{f}\left(\lambda_{k}\right)-f\left(\lambda_{k}\right)\right|=o_{P}(1) .
\end{aligned}
$$

\section{d) Local Bootstrap LB}

By Assumption $\mathcal{A} .4$ (ii) it holds

$$
\begin{aligned}
& \sup _{k}\left|\operatorname{var}^{*}\left(x^{*}(k)\right)-\pi f\left(\lambda_{k}\right)\right| \\
& \preceq \sup _{k}\left|\sum_{s \in \mathbb{Z}} p_{s, T} I(s+k)-2 \pi f\left(\lambda_{k}\right)\right|+\sup _{k}\left(\sum_{s \in \mathbb{Z}} p_{s, T}(x(k+s)+y(k+s))\right)^{2} \\
& =o_{P}(1)
\end{aligned}
$$


where $I(j)=x^{2}(j)+y^{2}(j)$, if $j$ is not a multiple of $T$ and $I(c T)=0$ for $c \in \mathbb{Z}$. Furthermore by Assumption $\mathcal{A} .4$ and $\mathcal{K} .1$ we have

$$
\begin{aligned}
& \sup _{k} \mathrm{E}^{*}\left(x^{*}(k)^{4}\right) \\
& \preceq \sup _{k}\left|\frac{1}{2} \sum_{s \in \mathbb{Z}} p_{s, T}\left(x(s+k)^{4}+y(s+k)^{4}\right)\right|^{4} \\
& +\sup _{k}\left(\frac{1}{2} \sum_{s \in \mathbb{Z}} p_{s, T}(x(s+k)+y(s+k))\right)^{4} \\
& \leqslant \sup _{k} \sum_{s \in \mathbb{Z}} p_{s, T} I^{2}(s+k)+o_{P}(1) \leqslant C+o_{P}(1) .
\end{aligned}
$$

Concerning $\mathcal{B}$.4 note first that $f$ is uniformly continuous (since it is continuous by $\mathcal{P} .1$ and periodic on $[0,2 \pi])$, hence

$$
\sup _{1 \leqslant k \leqslant N-T h_{T} \leqslant j \leqslant T h_{T}} \sup _{1 \leqslant k}\left|f\left(\lambda_{k+j}\right)-f\left(\lambda_{k}\right)\right|=o(1) .
$$

Denote now $\widetilde{\widetilde{x}}^{*}(j)=\widetilde{x}^{*}(j) / \sqrt{\pi f\left(\lambda_{\left.j+J_{j, T}\right)}\right.}$, where $J_{j, T}$ is the same random variable as in the definition of the Local Bootstrap. Then by (B.2) and Assumption $\mathcal{A}$.4 we get

$$
\begin{aligned}
& \sup _{1 \leqslant j \leqslant N} d_{2}^{2}\left(\mathcal{L}^{*}\left(x^{*}(j)\right), N\left(0, \pi f\left(\lambda_{j}\right)\right)\right) \\
& \preceq \sup _{j} d_{2}^{2}\left(\mathcal{L}^{*}\left(x^{*}(j)\right), \mathcal{L}^{*}\left(\widetilde{x}^{*}(j)\right)\right) \\
& \quad+\pi \sup _{l}\left|f\left(\lambda_{l}\right)\right| \sup _{j} d_{2}^{2}\left[\mathcal{L}^{*}\left(\widetilde{x}^{*}(j) / \sqrt{\pi f\left(\lambda_{j}\right)}\right), N(0,1)\right] \\
& \preceq \sup _{j}\left(\sum_{s \in \mathbb{Z}} p_{s, T}(x(j+s)+y(j+s))\right)^{2} \\
& \quad+\sup _{j} d_{2}^{2}\left[\mathcal{L}^{*}\left(\widetilde{x}^{*}(j) / \sqrt{\pi f\left(\lambda_{j}\right)}\right), \mathcal{L}^{*}\left(\widetilde{\widetilde{x}}^{*}(j)\right)\right]+\sup _{j} d_{2}^{2}\left(\mathcal{L}^{*}\left(\widetilde{\widetilde{x}}^{*}(j)\right), N(0,1)\right) \\
& \preceq o_{P}(1)+\sup _{1 \leqslant l \leqslant N-T h_{T} \leqslant k \leqslant T h_{T}} \frac{\left|f\left(\lambda_{l+k}\right)-f\left(\lambda_{l}\right)\right|}{f\left(\lambda_{l+k}\right) f\left(\lambda_{l}\right)} \sup _{j} \sum_{s \in \mathbb{Z}} p_{s, T} I(j+s) \\
& \quad+\sup _{s} d_{2}^{2}\left(\mathcal{L}^{*}\left(\widetilde{\widetilde{x}}^{*}(s)\right), N(0,1)\right) \preceq o_{P}(1) .
\end{aligned}
$$

The last line follows by Assumption $\mathcal{A}$.5. Note that convergence in the Mallows distance is equivalent to having convergence in distribution in addition to convergence of the first two moments. In this case the convergence is in all three cases uniformly in $s$ (confer Assumption $\mathcal{A} .4$ and $\mathcal{A}$.5). A similar argument (merging the triangular array into one single sequence in a smart way) as in the proof of Lemma 5.3 then also gives the uniform convergence in the Mallows distance. 
Proof of Corollary 4.1. We will verify that Assumptions $\mathcal{A}$.1, $\mathcal{A}$.2 as well as $\mathcal{A} .4$ remain true, which imply Assumptions $\mathcal{B} .2$ as well as $\mathcal{B}$.3. Concerning $\mathcal{B}$.4 we show that the Mallows distance between the bootstrap r.v. based on $\widehat{V}(\cdot)$ and the bootstrap r.v. based on $V(\cdot)$ converges to 0 .

We put an index $V$ resp. $\widehat{V}$ on our previous notation indicating whether we use $V$ or $\widehat{V}$ in the calculation of it, e.g. $x_{\widehat{V}}(j), x_{V}(j)$ resp. $y_{\widehat{V}}(j), y_{V}(j)$ denote the Fourier coefficients based on $\widehat{V}(\cdot)$ resp. $V(\cdot)$.

First note that by Theorem 4.4.1 in Kirch [29], it holds

$$
\left|\sum_{j=1}^{N}\left(\cos \left(t_{1} \lambda_{j}\right) \cos \left(t_{2} \lambda_{j}\right)+\sin \left(t_{1} \lambda_{j}\right) \sin \left(t_{2} \lambda_{j}\right)\right)\right| \leqslant \begin{cases}N, & t_{1}=t_{2}, \\ 1, & t_{1} \neq t_{2} .\end{cases}
$$

Furthermore denote by

$$
F_{T}(j):= \begin{cases}\sum_{t=1}^{T}(V(t)-\widehat{V}(t)) \cos \left(t \lambda_{j}\right), & 1 \leqslant j \leqslant N, \\ \sum_{t=1}^{T}(V(t)-\widehat{V}(t)) \sin \left(t \lambda_{j-N}\right), & N<j \leqslant 2 N .\end{cases}
$$

By (B.3), (4.1) and an application of the Cauchy-Schwarz inequality we obtain

$$
\begin{aligned}
& \left|F_{T}(j)\right| \leqslant \sum_{t=1}^{T}|V(t)-\widehat{V}(t)|=o_{P}\left(T \alpha_{T}^{-1 / 2}\right), \\
& \sum_{j=1}^{2 N} F_{T}^{2}(j) \\
& =\sum_{t_{1}=1}^{T} \sum_{t_{2}=1}^{T}\left(V\left(t_{1}\right)-\widehat{V}\left(t_{1}\right)\right)\left(V\left(t_{2}\right)-\widehat{V}\left(t_{2}\right)\right) \\
& \quad \times \sum_{j=1}^{N}\left(\cos \left(t_{1} \lambda_{j}\right) \cos \left(t_{2} \lambda_{j}\right)+\sin \left(t_{1} \lambda_{j}\right) \sin \left(t_{2} \lambda_{j}\right)\right) \\
& \preceq N \sum_{t=1}^{T}(V(t)-\widehat{V}(t))^{2}+\sum_{t_{1} \neq t_{2}}\left|\left(V\left(t_{1}\right)-\widehat{V}\left(t_{1}\right)\right)\left(V\left(t_{2}\right)-\widehat{V}\left(t_{2}\right)\right)\right| \\
& =o_{P}\left(T^{2} \alpha_{T}^{-1}\right) .
\end{aligned}
$$

With this definition we get

$$
\begin{aligned}
& x_{V}(j)-x_{\widehat{V}}(j)=\frac{1}{\sqrt{T}} \sum_{t=1}^{T}(V(t)-\widehat{V}(t)) \cos \left(-t \lambda_{j}\right)=T^{-1 / 2} F_{T}(j), \\
& y_{V}(j)-y_{\widehat{V}}(j)=T^{-1 / 2} F_{T}(N+j) .
\end{aligned}
$$


Since $a^{2}-b^{2}=-(a-b)^{2}+2 a(a-b)$ this implies

$$
\begin{aligned}
& I_{V}(j)-I_{\widehat{V}}(j) \\
& =-\frac{1}{T}\left(F_{T}^{2}(j)+F_{T}^{2}(N+j)\right)+2 \frac{1}{\sqrt{T}} x_{V}(j) F_{T}(j)+2 \frac{1}{\sqrt{T}} y_{V}(j) F_{T}(N+j) .
\end{aligned}
$$

We are now prepared to prove the assertions for the different bootstrap procedures. We start with the Wild Bootstrap because for it we only have to verify that Assumption $\mathcal{A} .1$ remains true. We start with the proof af b), since this is also a crucial step for the proof of a).

\section{b) Wild Bootstrap WB}

Recall that by assumption $K(x) \geqslant 0, \sup |K(x)|<\infty$ and

$$
\begin{aligned}
& \frac{2 \pi}{T h_{T}} \sum_{j \in \mathbb{Z}} K\left(\frac{2 \pi j}{T h_{T}}\right)=1+o(1), \\
& \sup _{\lambda \in[0,2 \pi]}\left|K_{h}(\lambda)\right|=O\left(h_{T}^{-1}\right) .
\end{aligned}
$$

Let

$$
p_{l, T}=\frac{K\left(\frac{2 \pi l}{T h_{T}}\right)}{\sum_{j \in \mathbb{Z}} K\left(\frac{2 \pi j}{T h_{T}}\right)}
$$

By an application of the Cauchy-Schwarz inequality and of the assertion in Lemma 5.1

$$
\begin{aligned}
& \sup _{k}\left|\widehat{f}_{V}\left(\lambda_{k}\right)-\widehat{f}_{\widehat{V}}\left(\lambda_{k}\right)\right|=\sup _{k} \sum_{j \in \mathbb{Z}} p_{k-j, T}\left|I_{V}(j)-I_{\widehat{V}}(j)\right| \\
& \preceq \frac{1}{h_{T} T^{2}} \sum_{j=1}^{2 N} F_{T}^{2}(j)+\sup _{k} \frac{1}{T^{1 / 2}} \sum_{j \in \mathbb{Z}} p_{k-j, T}\left(x_{V}(j) F_{T}(j)+y_{V}(j) F_{T}(N+j)\right) \\
& \preceq o_{P}\left(\frac{1}{h_{T} \alpha_{T}}\right)+\sup _{k} \frac{1}{T^{1 / 2}} \sqrt{\sum_{j \in \mathbb{Z}} p_{k-j, T} I_{V}(j) \frac{1}{h_{T} T} \sum_{j=1}^{2 N} F_{T}(j)^{2}} \\
& =o_{P}\left(\left(h_{T} \alpha_{T}\right)^{-1}\right)+o_{P}\left(\left(h_{T} \alpha_{T}\right)^{-1 / 2}\right)=o_{P}(1)
\end{aligned}
$$

for $\alpha_{T}=h_{T}^{-1}$. This shows that Assumption $\mathcal{A} .1$ remains true for $\{\widehat{V}(\cdot)\}$.

\section{a) Residual-Based Bootstrap RB}

From the argument above we already know that Assumption $\mathcal{A}$.1 remains true for $\{\widehat{V}(\cdot)\}$. We will now verify that Assumption $\mathcal{A}$.2 remain true in order to have $\mathcal{B} .2$ and $\mathcal{B} .3$. Recall Assumption P.3, thus similarly to above by (B.7) and 
(B.5) we get

$$
\begin{aligned}
& \frac{1}{N} \sum_{j=1}^{N} \frac{I_{V}(j)-I_{\widehat{V}}(j)}{f\left(\lambda_{j}\right)} \\
& =o_{P}\left(\alpha_{T}^{-1}\right)+\frac{1}{T^{3 / 2}} \sqrt{\sum_{j=1}^{N} \frac{I_{V}(j)}{f\left(\lambda_{j}\right)} \sum_{l=1}^{2 N} \frac{F_{T}^{2}(l)}{f\left(\lambda_{l}\right)}} \\
& =o_{P}\left(\alpha_{T}^{-1}+\alpha_{T}^{-1 / 2}\right)=o_{P}(1)
\end{aligned}
$$

by another application of the Cauchy-Schwarz inequality and by Assumption $\mathcal{A} .2$. Similarly

$$
\begin{aligned}
& \frac{1}{N} \sum_{j=1}^{N} \frac{\left(I_{V}(j)-I_{\widehat{V}}(j)\right)^{2}}{f^{2}\left(\lambda_{j}\right)} \\
& \preceq \frac{1}{T \alpha_{T}} \sum_{j=1}^{2 N} F_{T}^{2}(j)+\frac{1}{T^{2}} \sqrt{\sum_{j=1}^{N} \frac{I_{V}^{2}(j)}{f^{2}\left(\lambda_{j}\right)} \sum_{l=1}^{2 N} \frac{F_{T}^{4}(l)+F_{T}^{4}(N+l)}{f^{2}\left(\lambda_{l}\right)}} \\
& \preceq o_{P}\left(\frac{T}{\alpha_{T}^{2}}\right)+o_{P}\left(\frac{T^{1 / 2}}{\alpha_{T}}\right)=o_{P}(1)
\end{aligned}
$$

for $\alpha_{T}=O\left(T^{1 / 2}\right)$.

From this we get by $a^{2}-b^{2}=-(a-b)^{2}+2 a(a-b)$ and Assumption $\mathcal{A} .2$

$$
\begin{aligned}
& \frac{1}{N} \sum_{j=1}^{N} \frac{I_{V}^{2}(j)-I_{\widehat{V}}^{2}(j)}{f^{2}\left(\lambda_{j}\right)} \\
& \preceq \frac{1}{N} \sum_{j=1}^{N} \frac{\left(I_{V}(j)-I_{\widehat{V}}(j)\right)^{2}}{f^{2}\left(\lambda_{j}\right)}+\sqrt{\frac{1}{N} \sum_{j=1}^{N} \frac{I_{V}^{2}(j)}{f^{2}\left(\lambda_{j}\right)} \frac{1}{N} \sum_{l=1}^{N} \frac{\left(I_{V}(l)-I_{\widehat{V}}(l)\right)^{2}}{f^{2}\left(\lambda_{l}\right)}} \\
& =o_{P}(1) .
\end{aligned}
$$

Finally

$$
\begin{aligned}
& \frac{1}{N} \sum_{j=1}^{N} \frac{x_{V}(j)-x_{\widehat{V}}(j)+y_{V}(j)-y_{\widehat{V}}(j)}{\sqrt{f\left(\lambda_{j}\right)}} \preceq \frac{1}{T} \sqrt[3 / 2]{\sum_{j=1}^{N} \frac{1}{f\left(\lambda_{j}\right)} \sum_{l=1}^{2 N} F_{T}^{2}(l)} \\
& \preceq o_{P}\left(\alpha_{T}^{-1 / 2}\right)=o_{P}(1) .
\end{aligned}
$$

Finally we prove that $\mathcal{B} .4$ remains true. Looking carefully at the proof of this assertion for $\{V(\cdot)\}$ it is clear that it remains to prove that $d_{2}\left(\mathcal{L}^{*}\left(\widetilde{s}_{V, 1}^{*}\right), \mathcal{L}^{*}\left(\widetilde{s}_{\widehat{V}, 1}^{*}\right)\right) \rightarrow 0$, where $\widetilde{s}_{V, 1}^{*}$ is as $\widetilde{\widetilde{s}}_{1}^{*}$ in the proof of Theorem 4.1 and the one with $\widehat{V}$ corresponds to $\{\widehat{V}(\cdot)\}$ instead of $\{V(\cdot)\}$. With the same 
underlying random variable $U_{N}(1)$ we easily get

$$
\begin{aligned}
& d_{2}^{2}\left(\mathcal{L}^{*}\left(\widetilde{\widetilde{s}}_{V, 1}^{*}\right), \mathcal{L}^{*}\left(\widetilde{\widetilde{s}}_{\widehat{V}, 1}^{*}\right)\right) \leqslant \mathrm{E}^{*}\left(\widetilde{\widetilde{s}}_{V, 1}^{*}-\widetilde{\widetilde{s}}_{\widehat{V}, 1}^{*}\right)^{2} \\
& \preceq \frac{1}{T^{2}} \sum_{j=1}^{N} \frac{F_{T}^{2}(j)+F_{T}^{2}(N+j)}{f\left(\lambda_{j}\right)}=o_{P}\left(\alpha_{T}^{-1}\right)=o_{P}(1) .
\end{aligned}
$$

\section{c) Local Bootstrap LB}

By the exact same argument as for the Wild Bootstrap (in view of $\mathcal{K} .1$ ) we get

$$
\sup _{1 \leqslant k \leqslant N}\left|\sum_{j \in \mathbb{Z}} p_{j, T}\left(I_{V}(k+j)-I_{\widehat{V}}(k+j)\right)\right|=o_{P}\left(\left(\alpha_{T} h_{T}\right)^{-1}+\left(\alpha_{T} h_{T}\right)^{-1 / 2}\right)=o_{P}(1)
$$

for $\alpha_{T}=\left(T / h_{T}\right)^{1 / 2}$. Similarly to the proof for the Residual-Based Bootstrap we get

$$
\sup _{1 \leqslant k \leqslant N} \sum_{j \in \mathbb{Z}} p_{j, T}\left(I_{V}(k+j)-I_{\widehat{V}}(k+j)\right)^{2}=o_{P}\left(\frac{T}{h_{T} \alpha_{T}^{2}}+\frac{T^{1 / 2}}{h_{T}^{1 / 2} \alpha_{T}}\right)=o_{P}(1),
$$

which yields as above

$$
\sup _{1 \leqslant k \leqslant N} \sum_{j \in \mathbb{Z}} p_{j, T}\left(I_{V}^{2}(k+j)-I_{\widehat{V}}^{2}(k+j)\right)=o_{P}(1) .
$$

Finally

$$
\begin{aligned}
& \sup _{1 \leqslant k \leqslant N}\left|\sum_{j \in \mathbb{Z}} p_{j, T}\left(x_{V}(k+j)-x_{\widehat{V}}(k+j)+y_{V}(k+j)-y_{\widehat{V}}(k+j)\right)\right| \\
& =o_{P}\left(\left(\alpha_{T} h_{T}^{2}\right)^{-1 / 2}\right)=o_{P}(1) .
\end{aligned}
$$

Concerning $\mathcal{B} .4$ it holds similarly to above

$$
\sup _{j} d_{2}^{2}\left(\mathcal{L}^{*}\left(\widetilde{\widetilde{x}}_{V}^{*}(j)\right), \mathcal{L}^{*}\left(\widetilde{\widetilde{x}}_{\widehat{V}}^{*}(j)\right)\right)=o_{P}\left(\left(\alpha_{T} h_{T}\right)^{-1}\right)=o_{P}(1)
$$

which completes the proof.

\section{Appendix C: Proofs of Section 5}

Proof of Lemma 5.1. For a) see Theorem 2.1 in Robinson [53], which shows the result due to $\mathcal{K} .1$; b) is an easy consequence of Theorem 3.2 in Shao and $\mathrm{Wu}$ [55]. They even give a rate for the convergence of $\widehat{f}_{T}(\lambda)-\mathrm{E} \widehat{f}_{T}(\lambda)$. The only thing that still needs to be shown is

$$
\max _{\lambda \in[0,2 \pi]}\left|\mathrm{E} \widehat{f}_{T}(\lambda)-f(\lambda)\right|=o(1) .
$$


In fact it holds since by assumption $k(\cdot)$ is bounded (continuous and with compact support) and $k(0)=1$ as $T \rightarrow \infty$

$$
\begin{aligned}
& \left|\mathrm{E} \widehat{f}_{T}(\lambda)-f(\lambda)\right|=\left|\frac{1}{2 \pi} \sum_{j=-T}^{T} \frac{T-|j|}{T} \gamma(j) k(j h) e^{-i j \lambda}-\frac{1}{2 \pi} \sum_{j \in \mathbb{Z}} \gamma(j) e^{-i j \lambda}\right| \\
& \preceq \sum_{|j| \geqslant \sqrt{1 / h}}|\gamma(j)|+\frac{1}{T \sqrt{h}} \sum_{|j|<\sqrt{1 / h}}|\gamma(j)|+\sup _{|x| \leqslant \sqrt{h}}|k(x)-k(0)| \sum_{|j|<\sqrt{1 / h}}|\gamma(j)| \\
& =o(1) .
\end{aligned}
$$

Furthermore they use $I(c T)=T\left(\bar{V}_{T}-\mathrm{E} V(0)\right)^{2}$ but by $\mathcal{P} .2$ it holds $T\left(\bar{V}_{T}-\right.$ $\mathrm{E} V(0))^{2}=O_{P}(1)$ showing that this term is asymptotically negligible (confer also Remark 4.1).

Remark C.1. Shao and $\mathrm{Wu}[55]$ actually prove their results for the differentlooking estimator

$$
\widetilde{f}_{T}(\lambda)=\frac{1}{2 \pi} \sum_{j \in \mathbb{Z}} \widehat{R}(j) k(j h) \exp (-i k \lambda)=\frac{1}{2 \pi T} \sum_{t=0}^{T-1} I(t) K_{h}\left(\lambda-\lambda_{t}\right),
$$

where $\widehat{R}(j)=T^{-1} \sum_{l=1}^{T-|j|}(V(j)-\mathrm{E} V(1))(V(j+|l|)-\mathrm{E} V(1))$ and $K_{h}(\cdot)$ is as in (4.2). Hence by the $T$-periodicity of $I(j)$

$$
\begin{aligned}
& \widetilde{f}_{T}(\lambda)=\frac{1}{2 \pi h T} \sum_{t=0}^{T-1} I(t) \sum_{j \in \mathbb{Z}} K\left(\left(\lambda-\lambda_{t}+2 \pi j\right) / h\right) \\
& =\frac{1}{2 \pi h T} \sum_{j \in \mathbb{Z}} \sum_{t=0}^{T-1} I(t+j T) K\left(\left(\lambda-\lambda_{t+j T}\right) / h\right) \\
& =\frac{1}{2 \pi h T} \sum_{l \in \mathbb{Z}} I(l) K\left(\left(\lambda-\lambda_{l}\right) / h\right)=\widehat{f}_{T}(\lambda)+o(1)
\end{aligned}
$$

by Assumption $\mathcal{K} .1$, so that the Shao and Wu [55] estimator is identical to the one considered here.

Proof of Lemma 5.2. For the proof of a) we show that

$$
\begin{aligned}
& \sup _{1 \leqslant l, k \leqslant N}\left|\operatorname{cov}(x(l), x(k))-\pi f\left(\lambda_{k}\right) \delta_{l, k}\right| \rightarrow 0, \\
& \sup _{1 \leqslant l, k \leqslant N}\left|\operatorname{cov}(y(l), y(k))-\pi f\left(\lambda_{k}\right) \delta_{l, k}\right| \rightarrow 0 .
\end{aligned}
$$


Note that

$$
\begin{aligned}
& \operatorname{cov}(x(l), x(k))+\operatorname{cov}(y(l), y(k))=\operatorname{Re}\left(\frac{1}{T} \sum_{1 \leqslant j, s \leqslant T} e^{-i\left(j \lambda_{l}-s \lambda_{k}\right)} \operatorname{cov}(V(j), V(s))\right), \\
& \operatorname{cov}(x(l), x(k))-\operatorname{cov}(y(l), y(k))=\operatorname{Re}\left(\frac{1}{T} \sum_{1 \leqslant j, s \leqslant T} e^{-i\left(j \lambda_{l}+s \lambda_{k}\right)} \operatorname{cov}(V(j), V(s))\right) .
\end{aligned}
$$

Furthermore since $1 \leqslant l+k \leqslant T-1$ for all $1 \leqslant l, k \leqslant N$ it holds $\sum_{j=1}^{T} e^{-i j\left(\lambda_{l}+\lambda_{k}\right)}=$ 0 , hence

$$
\begin{aligned}
& \frac{1}{T} \sum_{1 \leqslant j, s \leqslant T} e^{-i\left(j \lambda_{l}+s \lambda_{k}\right)} \operatorname{cov}(V(j), V(s)) \\
& =\frac{1}{T} \sum_{j=1}^{T} e^{-i j\left(\lambda_{l}+\lambda_{k}\right)} \sum_{|h| \leqslant T-j} e^{-i h \lambda_{k}} \gamma(h) \\
& =\frac{1}{T} \sum_{j=1}^{T} e^{-i j\left(\lambda_{l}+\lambda_{k}\right)}\left(\sum_{|h| \leqslant T-j} e^{-i h \lambda_{k}} \gamma(h)-2 \pi f\left(\lambda_{k}\right)\right) \\
& \leqslant \frac{1}{T} \sum_{j=1}^{T} \sum_{|h|>T-j}|\gamma(h)| \leqslant T^{-1 / 2}+\sum_{|h|>T^{1 / 2}}|\gamma(h)|=o(1)
\end{aligned}
$$

uniformly in $l, k$ by the absolute summability of the autocovariance function (Assumption $\mathcal{P}$.1). Completely analogous we get for $l \neq k$, i.e. $\lambda_{l}-\lambda_{k} \neq 0$

$$
\frac{1}{T} \sum_{1 \leqslant j, s \leqslant T} e^{-i\left(j \lambda_{l}-s \lambda_{k}\right)} \operatorname{cov}(V(j), V(s))=o(1)
$$

uniformly in $l \neq k$. Finally,

$$
\begin{aligned}
& \frac{1}{T} \sum_{1 \leqslant j, s \leqslant T} e^{-i(j-s) \lambda_{k}} \operatorname{cov}(V(j), V(s))-2 \pi f\left(\lambda_{k}\right) \\
& =\sum_{|h|<T}\left(1-\frac{|h|}{T}\right) e^{-i h \lambda_{k}} \gamma(h)-2 \pi f\left(\lambda_{k}\right)=o(1)
\end{aligned}
$$

uniformly in $k$. Putting together (C.2) - (C.5) yields (C.1). Note that a refined version of (C.3)-(C.5) under the stronger assumption $\sum_{h}|h|^{\nu}|\gamma(h)|<\infty$ for some $\nu>0$ even gives the following uniform convergence rate

$$
\begin{cases}O\left(T^{-\nu}\right), & 0<\nu<1 \\ O(\log T / T), & \nu=1 \\ O\left(T^{-1}\right), & \nu>1\end{cases}
$$


Note that $\mathrm{E} x(k)=\mathrm{E} y(k)=0$, since $\frac{1}{T} \sum_{j=1}^{T} e^{-i j \lambda_{k}}=0$. Thus a simple application of the Markov-inequality yields by (C.1)

$$
\frac{1}{2 N} \sum_{j=1}^{N} \frac{x(j)}{\sqrt{f\left(\lambda_{j}\right)}}=o_{P}(1), \quad \frac{1}{2 N} \sum_{j=1}^{N} \frac{y(j)}{\sqrt{f\left(\lambda_{j}\right)}}=o_{P}(1),
$$

hence assertion a).

Since by Proposition 10.3.1 in Brockwell and Davis [5]

$$
\sup _{j}\left|\operatorname{E} I(j)-2 \pi f\left(\lambda_{j}\right)\right|=o(1)
$$

assertion b) follows from an application of the Markov inequality and (5.1).

Since $\mathrm{E} I^{2}(j)=\operatorname{var} I(j)+(\mathrm{E} I(j))^{2}$ it holds by $(5.1)$ and $($ C.7)

$$
\sup _{j}\left|\mathrm{E} I^{2}(j)-2\left(2 \pi f\left(\lambda_{j}\right)\right)^{2}\right|=o(1),
$$

hence by (5.2) and an application of the Markov inequality assertion c) follows.

Proof of Lemma 5.3. The proof is close to the proof of Corollary 2.2 in Shao and $\mathrm{Wu}[55]$ who prove an analogous result for the empirical distribution function of the periodograms. Denote by

$$
\widetilde{s}_{T}(j)= \begin{cases}\frac{x(j)}{\sqrt{\pi f\left(\lambda_{j}\right)}}, & 1 \leqslant j \leqslant N, \\ \frac{y(j-N)}{\sqrt{\pi f\left(\lambda_{j-N}\right)}}, & N+1 \leqslant j \leqslant 2 N .\end{cases}
$$

Theorem 2.1 in Shao and $\mathrm{Wu}[55]$ yields the uniform convergence of any linear combination of $\widetilde{s}(\cdot)$, i.e. for each fixed $p$

$$
\sup _{1 \leqslant j_{1}<j_{2}<\ldots<j_{p} \leqslant N ; c \in \mathbb{R}^{p} ;|c|=1}\left|P\left(\left(\widetilde{s}_{T}\left(j_{1}\right), \ldots, \widetilde{s}_{T}\left(j_{p}\right)\right)^{T} c \leqslant z\right)-\Phi(z)\right|=o(1) .
$$

First we will use an argument similar to one used by Freedman and Lane [19] to obtain the uniform convergence of vectors of $\widetilde{s}_{T}(\cdot)$. We will give the argument only for vectors of length 2 but the same holds true for length $p$. Precisely we will prove that

$$
\sup _{1 \leqslant j_{1} \neq j_{2} \leqslant N}\left|P\left(\widetilde{s}_{T}\left(j_{1}\right) \leqslant z_{1}, \widetilde{s}_{T}\left(j_{2}\right) \leqslant z_{2}\right)-\Phi\left(z_{1}\right) \Phi\left(z_{2}\right)\right|=o(1) .
$$

Now order the distributions of $\widetilde{S}_{T, j_{1}, j_{2}}=\left(\widetilde{s}_{T}\left(j_{1}\right), \widetilde{s}_{T}\left(j_{2}\right)\right), 1 \leqslant j_{1}<j_{2} \leqslant N$, $N \geqslant 1$, to form a single sequence $S_{t}=\left(S_{t}(1), S_{t}(2)\right)^{T}, t \geqslant 1$, in such a way that if $S_{t_{1}}$ corresponds to $\widetilde{S}_{T_{1}, j_{1,1}, j_{2,1}}$ and $S_{t_{2}}$ corresponds to $\widetilde{S}_{T_{2}, j_{1,2}, j_{2,2}}$, then $T_{1}<T_{2}$ implies that $t_{1}<t_{2}$. By Levy's continuity theorem and (C.8) it holds for each $z=\left(z_{1}, z_{2}\right)^{T}$ ( $\phi_{X}$ denotes the characteristic function of the random variable $X$ and $G_{1}, G_{2}$ are two independent standard normal random variables)

$$
\phi_{S_{t}}(z)=\phi_{\frac{z^{T} S_{t}}{|z|}}(|z|) \rightarrow \phi_{G_{1}}(|z|)=\phi_{\left(G_{1}, G_{2}\right)}(z) .
$$


Thus a second application of Levy'y continuity theorem yields

$$
\left|P\left(S_{t}(1) \leqslant z_{1}, S_{t}(2) \leqslant z_{2}\right)-\Phi\left(z_{1}\right) \Phi\left(z_{2}\right)\right|=o(1)
$$

and by definition of $S_{t}$ we get (C.9).

Define now $p_{j}(z)=P\left(\widetilde{s}_{T}(j) \leqslant z\right)$ and

$p_{j_{1}, j_{2}}(z)=P\left(\widetilde{s}_{T}\left(j_{1}\right) \leqslant z, \widetilde{s}_{T}\left(j_{2}\right) \leqslant z\right)$. Then it holds by (C.8) resp. (C.9)

$$
\begin{aligned}
& \left|\mathrm{E}\left(\sum_{j=1}^{N} w_{j, N} 1_{\left\{\tilde{s}_{T}(j) \leqslant z\right\}}\right)-\Phi(z)\right| \leqslant \sup _{l}\left|p_{l}(z)-\Phi(z)\right| \sum_{j=1}^{N} w_{j, N}=o(1), \\
& \left|\mathrm{E}\left(\sum_{j=1}^{N} w_{j, N} 1_{\left\{\tilde{s}_{T}(j) \leqslant z\right\}}\right)^{2}-\Phi^{2}(z)\right| \\
& \leqslant \sup _{j_{1} \neq j_{2}}\left|p_{j_{1}, j_{2}}(z)-\Phi^{2}(z)\right| \sum_{1 \leqslant j_{1} \neq j_{2} \leqslant N} w_{j_{1}, N} w_{j_{2}, N} \\
& \quad+\left(\sup _{l}\left|p_{l}(z)-\Phi(z)\right|+\left|\Phi(z)-\Phi^{2}(z)\right|\right) \sum_{j=1}^{N} w_{j, N}^{2}=o(1),
\end{aligned}
$$

which remains true uniformly in $s$ if we have weights $w_{j, N, s}$ depending on an additional parameter additionally with $\sup _{s} \sum_{j} w_{j, N, s}^{2} \rightarrow 0$. Since

$$
\begin{aligned}
& \mathrm{E}\left(\sum_{j=1}^{N} w_{j, N} 1_{\left\{\widetilde{s}_{T}(j) \leqslant z\right\}}-\Phi(z)\right)^{2} \\
= & \mathrm{E}\left(\sum_{j=1}^{N} w_{j, N} 1_{\left.\widetilde{s}_{T}(j) \leqslant z\right\}}\right)^{2}-\Phi^{2}(z) \\
& -2 \Phi(z)\left[\mathrm{E}\left(\sum_{j=1}^{N} w_{j, N} 1_{\left\{\widetilde{s}_{T}(j) \leqslant z\right\}}\right)-\Phi(z)\right]=o(1),
\end{aligned}
$$

we get both assertions by the Chebyshev inequality and the uniformity in $z$ follows from the continuity of $\Phi(z)$.

Proof of Lemma 5.4. The proof is very close to the proof of Theorem A.1 in Franke and Härdle [18] who essentially obtain rates for the situation of $\mathcal{A}$.2 (ii). Referring to the similarity of arguments, we only sketch the proof of the lemma. Let $a_{T}=h_{T} T^{-1 / 3}, m_{T}=\left\lfloor a_{T}^{-1}\right\rfloor$. Then the supremum in a) can be decomposed 
as follows, where $s_{l}=\left\lfloor l T / m_{T}\right\rfloor$

$$
\begin{aligned}
& \sup _{1 \leqslant k \leqslant N}\left|\sum_{j \in \mathbb{Z}} p_{j, T} x(k+j)\right| \\
& \leqslant \sup _{|l| \leqslant m_{T}}\left|\sum_{j \in \mathbb{Z}} p_{j, T} x\left(s_{l}+j\right)\right|+\sup _{|t-s| \leqslant T / m_{T}+1}\left|\sum_{j \in \mathbb{Z}} p_{j, T}(x(s+j)-x(t+j))\right| \\
& =O_{P}\left(h_{T}^{-1} T^{-1 / 3}\right) .
\end{aligned}
$$

The last line follows by the following two arguments: For the first summand it holds by Chebyshevs inequality, the assumptions on $K(\cdot)$ and $f(\cdot)$ as well as (5.4)

$$
\begin{aligned}
& P\left(h_{T} T^{1 / 3} \sup _{|l| \leqslant m_{T}}\left|\sum_{j \in \mathbb{Z}} p_{j, T} x\left(s_{l}+j\right)\right| \geqslant \epsilon\right) \\
& \leqslant \sum_{|l| \leqslant m_{T}} \frac{h_{T}^{2} T^{2 / 3}}{\epsilon^{2}} \operatorname{var}\left(\sum_{j \in \mathbb{Z}} p_{j, T} x\left(s_{l}+j\right)\right) \preceq m_{T} h_{T} T^{-1 / 3}=O(1) .
\end{aligned}
$$

For the second summand we get using $\mathcal{K} .1, \mathcal{K} .5$ and (5.3)

$$
\begin{aligned}
& \sup _{|t-s| \leqslant T / m_{T}+1}\left|\sum_{j \in \mathbb{Z}} p_{j, T}(x(s+j)-x(t+j))\right| \\
& =\sup _{|t-s| \leqslant T / m_{T}+1}\left|\sum_{j}\left(p_{j-s, T}-p_{j-t, T}\right) x(j)\right| \\
& \preceq \frac{1}{T h_{T}^{2} m_{T}} \sum_{j=1}^{T}|x(j)|=O_{P}\left(h_{T}^{-1} T^{-1 / 3}\right) .
\end{aligned}
$$

Analogous arguments yield the assertion for $y(\cdot)$ as well as for b) and c).

\section{Appendix D: Proofs of Section 6}

Proof of Theorem 6.1. It is sufficient to prove the assertion of Corollary 4.1 under $H_{0}$ as well as $H_{1}$, then the assertion follows from Theorem 3.1 as well as the continuous mapping theorem. By the Hájek-Renyi inequality it follows 
under $H_{0}$

$$
\begin{aligned}
& \frac{1}{T} \sum_{t=1}^{T}(V(t)-\widehat{V}(t))^{2}=\frac{\widehat{\tilde{k}}}{T}\left(\mu-\widehat{\mu_{1}}\right)^{2}+\frac{T-\widehat{\tilde{k}}}{T}\left(\mu-\widehat{\mu_{2}}\right)^{2} \\
& =\frac{\log T}{T}\left(\frac{1}{\sqrt{(\log T)}} \sum_{j=1}^{\tilde{\tilde{k}}}(V(t)-\mathrm{E}(V(t)))\right)^{2} \\
& \quad+\frac{\log T}{T}\left(\frac{1}{\sqrt{(\log T)(T-\widehat{\tilde{k}})}} \sum_{j=\widehat{\tilde{k}}+1}^{T}(V(t)-\mathrm{E}(V(t)))\right)^{2} \\
& =O_{P}\left(\frac{\log T}{T}\right),
\end{aligned}
$$

which yields the assertion of Corollary 4.1.

Under the alternative it holds analogously

$$
\begin{aligned}
& \frac{1}{T} \sum_{t=1}^{T}(V(t)-\widehat{V}(t))^{2} \\
& =\frac{\min (\widehat{\tilde{k}}, \tilde{k})}{T}\left(\mu_{1}-\widehat{\mu}_{1}\right)^{2}+\left|d+\mu_{j}-\widehat{\mu_{j}}\right|^{2} \frac{|\widehat{\tilde{k}}-\tilde{k}|}{T}+\frac{T-\max (\widehat{\tilde{k}}, \tilde{k})}{T}\left(\mu_{2}-\widehat{\mu}_{2}\right)^{2} \\
& =O_{P}\left(\max \left(\frac{\log T}{T}, \beta_{T}\right)\right),
\end{aligned}
$$

where $d=\mu_{1}-\mu_{2}$ and $j=2$ if $\widehat{\tilde{k}}<k$ and $d=\mu_{2}-\mu_{1}$ and $j=1$ otherwise, which yields the assertion of Corollary 4.1.

Proof of Theorem 6.2. Noting that $Y^{*}(k)=\sum_{j=1}^{k} V^{*}(j)$, the assertion follows from an application of Corollaries 4.1 and 3.1 as well as (6.4), since $V(t)-\widehat{V}(t)=$ $\left(\rho-\widehat{\rho}_{T}\right) Y(t-1)$. 